\title{
El uso sedentario de medios tecnológicos de pantalla: perfil sociodemográfico de los adolescentes españoles Sedentary use of screen-media: Sociodemographic profile of Spanish adolescents
}

\author{
Alexandra Valencia-Peris, José Devís-Devís y Carmen Peiró-Velert \\ Universidad de Valencia
}

\begin{abstract}
Resumen: El objetivo de este trabajo fue cuantificar el tiempo diario que pasan los adolescentes españoles utilizando sedentariamente los medios tecnológicos de pantalla y averiguar si existen diferencias significativas en función de las variables sociodemográficas sexo, curso y nivel socioeconómico. La muestra del estudio fue representativa de la población española escolar adolescente, ascendiendo a un total de 2983 participantes de 12 a 18 años de edad. Se administraron dos cuestionarios, uno de auto-informe (ASAQ) para conocer el tiempo que pasan diariamente los adolescentes utilizando los medios tecnológicos de pantalla de forma sedentaria, concretamente la televisión, reproductores de DVDs, el ordenador y los videojuegos; y el cuestionario FAS II, para conocer su nivel socioeconómico. Se llevó a cabo un MANOVA2x3x2, entre otros análisis, para conocer si existían diferencias significativas en función de las variables independientes del estudio. Los adolescentes españoles pasan una media de 3 horas diarias utilizando los medios tecnológicos de pantalla, siendo únicamente el 27.2\% quienes cumplen las recomendaciones actuales de uso ( $<2$ horas/día). Los resultados evidencian que, a nivel global, los chicos utilizan estos medios en mayor medida que las chicas, que hay un pico máximo en el uso a mitad de la adolescencia y que no existen diferencias entre el nivel socioeconómico de los adolescentes. En este sentido, conviene empezar a diseñar estrategias de limitación del tiempo sedentario vinculado al uso de estos medios en los adolescentes, con especial hincapié en los chicos y en aquéllos que se encuentran al final de la Educación Secundaria Obligatoria.
\end{abstract}

Palabras clave: sedentarismo; medios tecnológicos de pantalla; jóvenes; sexo; curso; nivel socioeconómico.

\begin{abstract}
The aim of this study was to quantify the adolescents' sedentary use of screen time and find out if there were significant differences depending on the sociodemographic variables sex, school grade and socioeconomic status. Participants comprised a representative sample of Spanish adolescents ( $\mathrm{n}=2983$ ) aged 12 to 18 years old. Two questionnaires were administered: 1 ) a self-report questionnaire (ASAQ) to know the daily mean time adolescents engaged in sedentary use of screen media, in particular in TV, DVD player, computer and videogames; and 2) the FAS II questionnaire, to know their socioeconomic status. A 2x3x2 MANOVA was carried out, among other analyses, to determine if there were significant differences between the independent variables of the study. Spanish adolescents spent 3 hours on average daily engaging in screen media devices, being only the $27.2 \%$ the adolescents who met the current media recommendations ( $<2$ hours/day). Results show that, in general, boys spent more time than girls on screen media use, there is a high peak of use at the middle of adolescence and there are not significant differences among the different socioeconomic levels. In this sense, we recommend starting the design of strategies in order to limit the sedentary screen time in adolescents, focusing mainly on boys and in those who are finishing Secondary School studies.
\end{abstract}

Key words: sedentarism; screen media; youth; sex; school grade; socioeconomic status.

\section{Introducción}

Los estudios realizados hasta el momento sobre los riesgos de seguir un estilo de vida sedentario han provocado un aumento de la preocupación por la salud de las personas que viven en sociedades desarrolladas. Entre los principales riesgos para la salud que comporta seguir un estilo de vida sedentario se encuentran la obesidad, la hipertensión, enfermedades coronarias, diabetes tipo 2 y otras enfermedades crónicas que provocan un aumento en las tasas de mortalidad y una menor esperanza de vida (Del Pozo-Cruz, Del Pozo-Cruz, González \& Alfonso, 2012; Tremblay, Colley, Saunders, Healy \& Owen, 2010).

Según los expertos, las conductas sedentarias son multifacéticas y podrían incluir acciones o conductas que se realizan en el trabajo o en la escuela, en casa, utilizando un medio de transporte o en el tiempo libre (The Sedentary Behaviour and Obesity Expert Working Group, 2010). Sin embargo, cabe señalar que, cuando hablamos de niños y adolescentes, existen una serie de conductas sedentarias consideradas clave y que incluyen el tiempo dedicado al uso de los medios tecnológicos de pantalla(UMTP), tales como ver la televisión (TV), utilizar el ordenador y jugar a videojuegos. Éstas abarcan la mayor parte del tiempo sedentario de los jóvenes, excluyendo las horas que pasan sentados en horario escolar (Australian Bureau of Statistics, 2006). Es por ello que la American Academy of Pediatrics (AAP) ya se ha posicionado en varias ocasiones sugiriendo que los niños y adolescentes no deberían estar más

Fecha recepción: 04-01-14- Fecha envío revisores: 06-01-14- Fecha de aceptación: 04-03-14 Alexandra Valencia Peris

Av. Blasco Ibáñez, 21

46010 Valencia

alexandra.valencia@uv.es de dos horas diarias dedicados al UMTP y se debería prohibir su uso a menores de 2 años de edad (AAP, 2001; Council on Communication and Media, 2013). En este sentido, ya existen numerosos estudios que han encontrado asociaciones entre un uso excesivo de los medios tecnológicos y diversos riesgos para la salud de los niños y adolescentes. Por ejemplo, existe mayor probabilidad de que padezcan sobrepeso u obesidad(Hancox, Milne \& Poulton, 2004), síndrome metabólico (Mark \& Janssen, 2008), dispongan de una baja condición física (Hardy, Dobbins, Denney-Wilson, Okely \& Booth, 2009) o sufran trastornos emocionales y psicológicos (Moncada \& Chacón, 2012; Tremblay et al., 2010).

A nivel internacional son varias las investigaciones que se han llevado a cabo para cuantificar el UMTP por parte de los niños y jóvenes. Destacan diversas revisiones y estudios que comparan muestras de diferentes países a gran escala (Currie, Gabhainn et al., 2008; Ianotti et al., 2009; Te Velde et al., 2007). Anivel global se ha observado que los chicos realizan un mayor UMTP que las chicas. Aunque no existen grandes diferencias de género en cuanto al tiempo diario que pasan viendo $T V$, los resultados varían si hacemos referencia al uso del ordenador 0 al de los videojuegos, donde son los chicos quienes dedican más tiempo en comparación con las chicas (Te Velde et al., 2007; Thibault, Contrand, Saubusse, Baire \& Maurice-Tison, 2010). Por otro lado, parece existir un incremento del UMTP a medida que aumenta la edad de los jóvenes. Y en relación con el nivel socioeconómico(NSE), existen resultados inconsistentes.

En el contexto español ya existen varios trabajos que han abordado esta temática desde principios del siglo XXI. Por ejemplo los resultados de la Encuesta Nacional de Salud (ENS, 2006) indicaban que un 92.3\% de los chicos y un $91 \%$ de las chicas de 10 a 15 años veían la TV/vídeo/ DVD todos o casi todos los días de la semana. Los porcentajes relativos a la categoría 'Ver la TV/vídeos/DVDs' 3 o más horas diarias se 
incrementaban de un $19.4 \%$ a un $46 \%$ de entre semana a fin de semana para los chicos y de un $18.7 \%$ a un $46 \%$ para las chicas. En relación con la utilización de videojuegos, el ordenador o internet, los resultados mostraban una proporción del $75.5 \%$ de chicos y del $58.4 \%$ de las chicas de 10 a 15 años que los empleaban todos o casi todos los días. Quienes se encontraban más representados en la categoría de uso de 3 horas diarias o más, tanto entre semana como en fin de semana, eran los chicos en comparación con las chicas (8.5\% frente al $5.9 \%$ entre semana y 29.9\% frente al 20.8\% en fin de semana). Otra investigación realizada con muestra española es el estudio AVENA, del cual se recabaron datos procedentes de 2859 adolescentes de edades comprendidas entre los 13 y los 18 años. Según Rey-López et al. (2010) los adolescentes españoles presentaban diferencias en cuanto a las conductas sedentarias evaluadas según el sexo, la edad y el NSE. Mientras que no se daban diferencias en cuanto a ver la TV, los hombres jugaban más a videojuegos que las mujeres entre semana, y las mujeres pasaban más tiempo estudiando que los hombres. Otro trabajo que también derivó de este proyecto es el de Vicente-Rodríguez et al. (2008) en el cual se señalaba que los porcentajes de adolescentes que veían la TV más de 4 horas diarias eran mayores para los sujetos con sobrepeso (4.8\% y 5.9\% para hombres y mujeres, respectivamente) que para los jóvenes con normopeso (3.4\% para hombres y 2.9\% para mujeres). Hernández y Martínez (2007) también aportaron información relacionada tras realizar un estudio similar en el que constataron que los alumnos de $2^{\circ} \mathrm{de} E S O$ eran los que más veían la TV (1.03 h/día) seguidos por los de $4^{\circ}$ de ESO, siendo los alumnos de $4^{\circ}$ de Primaria quienes menos la veían. En cuanto a los juegos de ordenador o consola, eran los más jóvenes quienes más los utilizaban y las conductas sedentarias de chatear o navegar en internet experimentaban un aumento de la dedicación a medida que aumentaba la edad. Por último, cabe destacar el trabajo de Ramos, Rivera, Moreno y Jiménez-Iglesias (2012) con datos extraídos de la HBSC (Health Behavior in School-Aged Children Survey)-2006, y en el que indicaban que los adolescentes dedicaban una media diaria de 2.37 horas a verTV, 1.09 horas a jugar al ordenador o la consola y 1.11 horas al día a chatear, navegar por internet, mandar e-mails o hacer los deberes en el ordenador. Únicamente se encontraron diferencias por sexo en el tiempo de uso del ordenador con un fin social o académico y no lúdico, donde eran los chicos los que más tiempo le dedicaban.

En la actualidad, el ritmo al que avanzan las tecnologías de la información y el desarrollo de nuevos dispositivos de pantalla electrónicos es vertiginoso. Por ello, se requiere de estudios actualizados sobre las conductas sedentarias que van adoptando los y las adolescentes, quienes son considerados usuarios potenciales de los mismos. Por ello, el objetivo de este trabajo fue cuantificar el tiempo diario que pasaba una muestra representativa de adolescentes españoles utilizando estos medios tecnológicos y averiguar si existían diferencias significativas en función de las variables sociodemográficas sexo, curso y NSE.

\section{Metodología}

\section{Participantes}

De los 3095 escolares que participaron en el estudio y entregaron el consentimiento informado se descartaron 112 por haber cumplimentado erróneamente el cuestionario o por falta de información en las variables de estudio. La muestra final del estudio estuvo compuesta por 2983 escolares adolescentes de entre 12 y 18 años, es decir de $1^{\circ}$ de ESO a $2^{\circ}$ de Bachillerato (BACH), siendo representativa de la población adolescente escolarizada en contexto español. Del total de participantes, un $50.7 \%(n=1514)$ fueron chicas y un $49.3 \%$ fueron chicos $(\mathrm{n}=1469)$.

El tipo de muestreo fue polietápico. Como unidades primarias de muestreo se tomaron los códigos postales de los municipios de todo el territorio español (seleccionados de forma aleatoria para cada una de las seis zonas geográficas definidas y también utilizadas en el estudio de Serra et al., 2003). Posteriormente se hizo un sorteo aleatorio sobre las unidades secundarias de muestreo, es decir, sobre los centros públicos y privados de los municipios en cuestión. Finalmente, se eligió a los participantes por cuotas cruzadas de sexo y curso (de $1^{\circ}$ de ESO a $2^{\circ}$ de $\mathrm{BACH}$ ). Para determinados análisis se estableció una clasificación en función del ciclo educativo ( $1^{\text {er }}$ Ciclo ESO, $2^{\circ}$ Ciclo ESO y Ciclo de $\mathrm{BACH})$.

\section{Procedimiento}

El estudio contó con la aprobación del Comité de ética de la Universidad de Valencia y con la autorización de aquellas comunidades autónomas que requerían un permiso para investigar en centros educativos. El trabajo de campo se realizó durante el último trimestre de 2010. Asimismo, se solicitó un consentimiento informado a cada uno de los participantes que debía ser firmado por sus padres, madres o tutores y también se les garantizó el anonimato.

\section{Instrumentos}

Para conocer el nivel socioeconómico (NSE) de los participantes se utilizó la Family Affluence Scale II (FAS II) (Currie, Molcho et al., 2008). Esta medida refleja los recursos materiales de la familia, es decir, los bienes específicos que pueden permitirse sus miembros con los ingresos familiares (los ítems incluyen número de coches familiares, de ordenadores, de vacaciones y la posesión o no de habitación propia). La FAS II ha sido validada en investigaciones previas y los resultados confirman que es un indicador válido cuando se utiliza con adolescentes, tal y como señalan investigaciones en las que se comparan varios países (Borracchino etal. 2009; Von Rueden, Gosch, Rajmil, Bisegger \& RavensSieberer, 2006). La suma de las puntuaciones de cada uno de los cuatro ítems resulta en una cifra comprendida entre los 0 y los 9 puntos. De acuerdo con el protocolo internacional, se utilizó una escala ordinal que daba lugar a 3 categorías en función de la puntuación total calculada: NSE bajo, medio y alto. Para los análisis esta variable se recodificó en NSE bajo-medio y alto.

Con el objetivo de averiguar el patrón de actividades sedentarias se empleó una versión ampliada del ASAQ (Adolescent Sedentary Activity Questionnaire), el cual tiene una validez y una fiabilidad aceptables (Hardy, Bass \& Booth, 2007; Hardy, Booth \& Okely, 2007) y ha sido empleado en múltiples ocasiones en población adolescente (Dewar et al., 2013; Nihill, Lubans \& Plotnikoff, 2012). ElASAQ es un cuestionario que registra las actividades sedentarias realizadas fuera del horario escolar (en horas y minutos diarios) durante una semana habitual. Para este estudio únicamente se ha contemplado el uso de los siguientes medios tecnológicos de pantalla (UMTP): la televisión/reproductor DVD, el ordenador (para jugar y/o comunicarse) y la videoconsola.

\section{Análisis estadístico de los datos}

Tras la codificación, depuración y agrupación de los datos, se realizó una exploración de datos que confirmó una distribución asimétrica de las variables de UMTP. Una vez eliminados algunos de los valores atípicos por considerar imposible su realización en el periodo de un día, se optó por una transformación de las variables por Raíz Cuadrada (Booth, Okely, Chey \& Bauman, 2002; Pate et al., 2008), la cual supone una transformación mínima de los datos según la escalera de potencias de Tukey (1977). Este procedimiento permite obtener una distribución normal de los datos, evitando de esta manera problemas con la asunción de homogeneidad de varianza. Tras la recodificación de las variables, necesaria para poder aplicar pruebas paramétricas a los datos de nuestro estudio, los valores correspondientes a la asimetría y curtosis se suavizaron respecto a los encontrados en un principio. A partir de aquí se realizaron los análisis correspondientes tanto con las variables transformadas como con las originales para una mejor comprensión de los resultados.

En primer lugar se realizó un análisis descriptivo de las variables de estudio junto con un análisis de correlaciones bivariadas entre las variables dependientes. Para conocer si existían diferencias significativas en el tiempo dedicado al UMTP, se realizó un MANOVA 2 (sexo) x 3 (ciclo) x 2 (NSE) en el que se incluyeron como variables dependientes el 
tiempo dedicado a ver TV/reproductor DVD, el tiempo que se pasa utilizando el ordenador, el tiempo que se dedica a jugar a videojuegos y el tiempo global de UMTP. Asimismo, se llevó a cabo unANOVAde un factor para conocer la evolución del UMTP en función del curso académico. Para realizar estas operaciones estadísticas se utilizó el programa SPPS v.19.0.

\section{Resultados}

Valores globales de uso de medios tecnológicos de pantalla

El tiempo medio de UMTP por parte de los adolescentes españoles fue de 3 horas diarias ( $D T=1.52)$. En este sentido, únicamente el $27.2 \%$ de los adolescentes españoles cumple con las recomendaciones actuales de UMTP ( $<2$ horas/día). En la Tabla 1 se observa el número de participantes que respondió a cada tipo de conducta sedentaria (CS). La CS a la que más tiempo dedicaban era ver programas de TV, vídeos o DVDs (aproximadamente 1 hora y media), seguida por el uso del ordenador (alrededor de 1 hora y 10 minutos) y jugar a videojuegos pasivos enúltimo lugar (cerca de un cuarto de hora al día). En cualquiera de las tres actividades sedentarias se encontraron jóvenes que no las practicaban a diario. Por otro lado, encontramos tres adolescentes que informaban ver la TV más de 7 horas diarias, tres adolescentes que utilizaban el ordenador más de 7 horas diarias y dos casos que jugaban a los videojuegos más de 5 horas diarias. Exceptuando estos valores atípicos, el resto de participantes presentaba valores medios aceptables si comparamos éstos con el resto de actividades diarias.

Sellevaron a cabo correlaciones bivariadas para conocer si aquellos adolescentes que más utilizaban un medio tecnológico también eran más

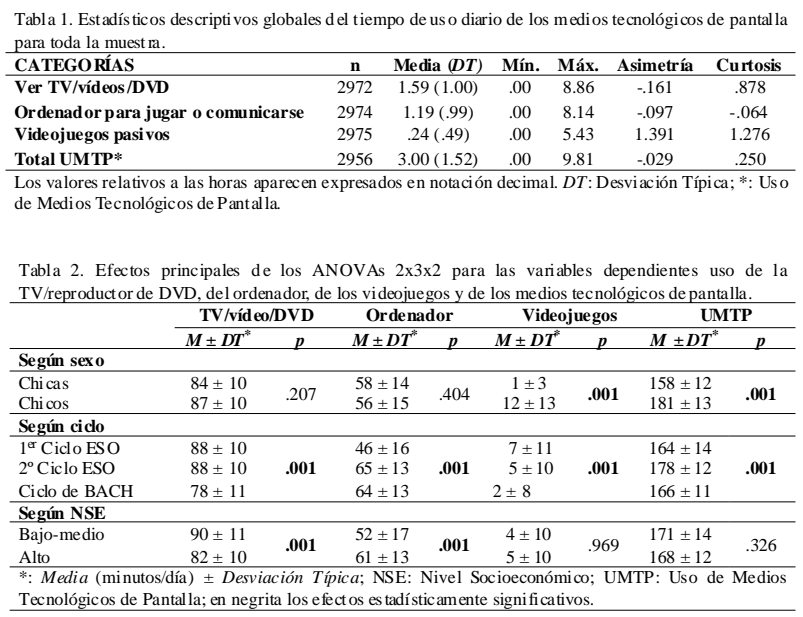

propensos a utilizar cualquier otro. Únicamente se encontró una correlación significativa y positiva, aunque baja, entre ver TV/vídeos/DVDy utilizar los videojuegos $(r=.145 ; p<.05)$.

Valores de uso de medios tecnológicos de pantalla según variables sociodemográficas

Según el sexo

El análisis multivariado reveló un efecto principal significativo referente al sexo (lambda de Wilks $=.825 ; F_{(42889)}=153.207 ; p<.001 ; n^{2}=.175$ ), al ciclo (lambda de Wilks $=.952 ; F_{(8.578)}=17.879 ; p<.001 ; n^{2}=.024$ ) y al NSE (lambda de Wilks $=.982 ; F_{(4.2889)}=12.888 ; p<.001 ; n^{2}=.018$ ). Cabe destacar el tamaño del efecto de la variable sexo, que explica un $17.5 \%$ de la varianza del UMTP. Por otro lado, no se encontraron efectos de interacción. El MANOVA mostró diferencias en la combinación lineal de las cuatro variables dependientes, tal y como revelan los ANOVAS de continuación, los cuales pueden observarse en la Tabla 2.

Se aprecian diferencias significativas en función del sexo para el UMTP global $\left(F_{(1.2892)}=39.666 ; p<.001 ; n^{2}=.014\right)$ y el uso de los videojuegos $\left(F_{(1.282)}=605.153 ; p<.001 ; n^{2}=.173\right)$ dedicando, en ambos casos, mayor tiempo los chicos que las chicas. Por otra parte, los chicos dedicaban una media de 3 horas diarias al uso global de los medios tecnológicos de pantalla, mientras que las chicas los utilizaban unas 2 horas y media al día. Y en cuanto al uso de videojuegos pasivos, los chicos pasaban 12 minutos al día mientras que las chicas prácticamente no los utilizaban.

Cabe resaltar que el hecho de que los chicos jugaran más a los videojuegos que las chicas, cuyo tamaño del efecto $\left(n^{2}=.173\right)$ explica un 17.3\% de la varianza de los datos, afectaba a las diferencias significativas encontradas en el UMTP global entre chicas y chicos. Es decir, al no encontrar diferencias significativas en función del sexo en el tiempo dedicado a ver la TV o a utilizar el ordenador y únicamente observarlas en el patrón de conducta asociada al uso de videojuegos, se entiende que esa sea la conducta que determina un mayor UMTP a nivel global por parte de los chicos.

\section{Según el curso y el ciclo}

En cuanto al ciclo, se observaron diferencias significativas tanto en el UMTP global $\left(F_{(22892)}=9.010 ; p<.001 ; n^{2}=.006\right)$, como en el uso aislado de la TV $\left(F_{(2.2892)}^{(2.2892)}=7.939 ; p<.001 ; n^{2}=.005\right)$, del ordenador $\left(F_{(2.2892)}=36.500 ; p<.001 ; n^{2}=.025\right)$ y de los videojuegos $\left(F_{(1.2892)}=26.600 ; p<.001 ; n^{2}=.018\right)$. Las pruebas post-hoc de Bonferroni revelaron que los estudiantes de $2^{\circ}$ Ciclo de ESO utilizaban más los medios tecnológicos a nivel global (2 horas y 38 minutos diarios) que el resto de ciclos $(p<.05)$. Por otro lado, el tiempo diario dedicado a ver TV por parte de los adolescentes que cursaban el Ciclo de BACH (1 hora y 18 minutos) era significativamente menor al del resto de ciclos, quienes la veían 10 minutos más al día ( $p<.05)$. Asimismo, el uso que hacían del ordenador los adolescentes de $1^{\text {er }}$ Ciclo de ESO (46 minutos), es decir, los más jóvenes, difería significativamente del resto de ciclos, quienes lo utilizaban más de 1 hora diaria. En cuanto al uso de videojuegos, existen diferencias significativas entre todos los ciclos $(p<.05)$, siendo los que más lo utilizaban los más jóvenes ( $1^{\text {er }}$ Ciclo de la ESO) ( 7 minutos diarios) y disminuyendo esta conducta a medida que el ciclo aumenta. Con el objetivo de observar la evolución en el UMTP más detalladamente se realizó un ANOVA de un factor para cada medio tecnológico. Los resultados revelaron diferencias significativas en el tiempo dedicado a ver TV $\left(F_{(5.2971)}=5.970 ; p<.001\right)$, a utilizar el ordenador $\left(F_{(52973)}=25.740 ; p<.001\right)$ y al tiempo que se dedica a los videojuegos $\left(F_{(5.2974)}=9.884 ; p<.001\right)$ según el curso académico de los adolescentes (Figura 1). Observamos diversos aspectos a destacar en lo referente a las pruebas post-hoc de Bonferroni. En relación con la TV/vídeos/DVD se observa un aumento significativo del tiempo dedicado a ver TV de $1^{\circ}$ a $2^{\circ}$ de ESO de unos 11 minutos aproximadamente $(p<.05)$ y, a partir de entonces, empieza a disminuir de forma paulatina el tiempo que se dedica diariamente hasta $2^{\circ} \mathrm{deBACH}$ (quienes dedican 1 hora y cuarto), media que difiere significativamente de las correspondientes a $2^{\circ}, 3^{\circ}$ y $4^{\circ}$ de ESO (más de 1 hora y 20 minutos) ( $p<.05$ ). Por otro lado, los jóvenes que más tiempo diario dedicaban al ordenador eran los que cursaban $1^{\circ}$ de BACH, es decir, aquéllos con 17 años, con una 1 hora y 7 minutos diarios. A este curso, le siguen los adolescentes de $3^{\circ}$ y $4^{\circ}$ de ESO, quienes dedicaban 2 minutos menos que los de $1^{\circ}$ de BACH. Es interesante también el aumento exponencial de esta conducta sedentaria de uso del ordenador de los que cursan $1^{\circ}$ de ESO a los que cursaban $2^{\circ}$ y $3^{\circ}$ de ESO (en 20 y 30 minutos más al día), siendo los adolescentes que

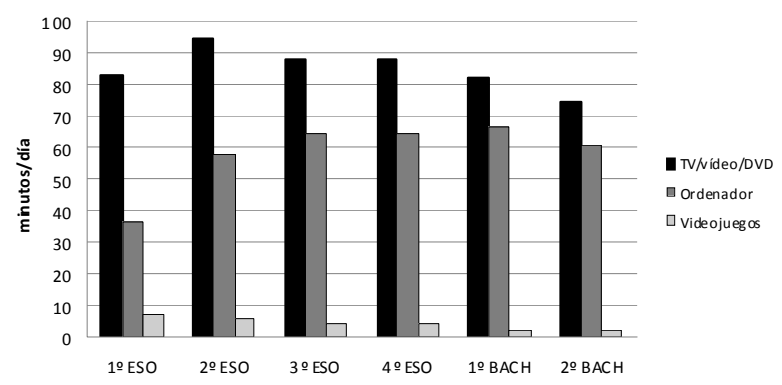

Figura 1. Tiempo dedicado al cada uno de los medios tecnológi cos de pantalla en fun ción del curso académico. 
cursaban $1^{\circ}$ de ESO los que mostraron diferencias significativas en cuanto a un menor uso respecto al resto de cursos $(p<.01)$. Y en el uso de videojuegos el patrón de conducta difiere de los otros dos medios estudiados. Se ha visto una disminución progresiva del tiempo dedicado a esta conducta conforme aumenta el curso. Concretamente eran los jóvenes de $1^{\circ}$ y los de $2^{\circ}$ de ESO los que diferían significativamente de los que cursaban $1^{\circ}$ y $2^{\circ}$ de BACH $(p<.05)$, y los de $3^{\circ}$ y $4^{\circ}$ de ESO los que dedicaban significativamente más tiempo que los adolescentes de $2^{\circ} \mathrm{de}$ BACH ( $p<.05)$. Curiosamente, en ningún curso se pasaba de los 10 minutos al día, a lo cual creemos que influencia el escaso uso que realizan de este medio tecnológico las chicas adolescentes.

\section{Según el Nivel Socioeconómico}

El NSE es otra de las variables que también mostró diferencias significativas en relación con el uso de la TV $\left(F_{(1.2892)}=7.939 ; p<.001\right.$; $\left.n^{2}=.005\right)$ y del ordenador $\left(F_{(1.2892)}=18.075 ; p<.001 ; n^{2}=.006\right)$. Concretamente, eran los adolescentes con un perfil de NSE bajo-medio los que veían más TV (8 minutos más) y utilizaban menos el ordenador (9 minutos menos) que aquéllos con un NSE alto. No se encontraron diferencias en función del NSE de los adolescentes ni en la dedicación diaria a los videojuegos ni en el tiempo que pasaban al día utilizando los medios tecnológicos de pantalla a nivel global.

\section{Discusión}

En cuanto al UMTP, y contrastando nuestros resultados con los aportados por otros estudios realizados con muestra adolescente española, apreciamos que, a nivel global, la media dedicada al UMTP por los participantes de nuestro estudio (3 horas diarias) es mayor a la aportada por el trabajo de Devís-Devís, Peiró-Velert, Beltrán-Carrillo y Tomás (2009) que otorgaba 2 horas diarias al UMTP. Al comparar nuestros resultados con los de otros países podemos señalar que la media de UMTP de nuestra muestra es muy similar a la proporcionada por Hardy et al. (2009) para adolescentes australianos, a la de Aires et al. (2010) para adolescentes portugueses (sin contar el tiempo dedicado a videojuegos) y a los adolescentes del sur, del norte y del Oeste de Europa (Ianotti et al., 2009). Nuestro resultado de 3 horas diarias sería inferior a la media aportada por el estudio de Thibault et al. (2010) con adolescentes franceses (3.57 horas/día) y a la de adolescentes estadounidenses y canadienses, que son los que más tiempo dedican al UMTP empleando 6.71 horas/día y 7.80 horas/día respectivamente(Leatherdale \&Ahmed, 2011; Rideout, Foehr \& Roberts, 2010).

Al diferenciar por tipo de UMTP, los resultados correspondientes al tiempo dedicado a ver TV en nuestra muestra ( $M=1.59$ horas/día) son similares a los del estudio de Nuviala, Munguía, Fernández y Ruiz (2009), quienes lo estimaban en 1.89 horas/día de media. No obstante, el informe relativo a la encuesta HBSC de 2006 elaborado por Moreno et al. (2011) otorgaba una media de 2.28 horas diarias dedicadas a ver TV por los adolescentes españoles, el de Ramos et al. (2012) estimaba una media diaria de 2.37 horas y el estudio de Moral et al. (2012) informaba de 2.24 horas diarias empleadas por los adolescentes andaluces, cifras bastante superiores a la de nuestro estudio.

Si confrontamos nuestros resultados con los de otros países, vemos que los adolescentes de nuestro estudio ven más la TV a diario que los adolescentes chinos (Li, Dibley, Sibbritt, Zhou \& Yan, 2007) pero menos que los adolescentes europeos a nivel global, que dedican más de 2 horas diarias (Aires et al., 2010; Patriarca, Di Giuseppe, Albano, Marinelli \& Angelillo, 2010; Te Velde et al., 2007), que los adolescentes brasileños, quienes dedican más de 3 horas (Romero et al., 2011), o que los adolescentes norteamericanos que dedican entre 2 y más de 4 horas al día, según diversos estudios (Beets \& Foley, 2010; Norman, Schmidt, Sallis, Calfas \& Patrick, 2005; Rideout et al., 2010). Marshall, Gorely y Biddle (2006), en una revisión de 99 trabajos publicados entre 1949 y 2004, proporcionaron información relativa al tiempo dedicado al UMTP por los adolescentes a nivel internacional y establecieron una media de 2.83 horas diarias a ver TV por parte de los adolescentes. En este sentido, pese a que los adolescentes de nuestro estudio ven la TV una hora y media al día, aún están lejos de las casi 3 horas, de media, encontradas en el citado estudio de revisión.

En cuanto al uso del ordenador ( $M=1.19$ horas/día), nuestros resultados son similares a los de Nuviala et al. (2009) (1.27 horas/día) y a los de Ramos et al. (2012) (1.11 horas/día), ambos realizados con muestra española. Mientras que nuestra media quedaría por encima de la otorgada por Marshall et al. (2006) de 0.95 horas diarias y por la dedicada por los adolescentes brasileños ( 0.28 horas/día) (Dumith, Hallal, Menezes \&Araújo, 2010), al contrastarla con otros países con medias superiores vemos que los adolescentes españoles no utilizan tanto el ordenador como lo hacen los italianos (Patriarca et al., 2010), los norteamericanos (Beets \& Foley, 2010; Rideout et al., 2010 y), los portugueses (Aires et al., 2010) o los chinos (Ho \& Lee, 2001). Estos últimos lo utilizan una media de 2.28 horas diarias. $\mathrm{Y}$ en relación con el uso de videojuegos ( $M=.24$ horas/día), nuestros resultados son parecidos a la media aportada por el estudio de Devís-Devís et al. (2009) con muestra española ( $M=.27$ horas/día dedicadas al ordenador y los videojuegos) y quedaría bastante por debajo del tiempo dedicado por los adolescentes italianos ( $M=1.65$ horas/día) (Patriarca et al., 2010) y por los norteamericanos, quienes sobrepasan la hora diaria (Beets \& Foley, 2010; Rideout et al., 2010). Los tiempos menores de uso de ordenador y videojuegos de los adolescentes españoles comparados con los europeos podrían estar relacionados con el retraso en la introducción de estos medios tecnológicos en comparación con países del norte de Europa o con Estados Unidos o Japón.

En cuanto al cumplimiento de las recomendaciones de UMTP (menos de 2 horas diarias) por parte de los adolescentes españoles de nuestra muestra, la cifra encontrada es bastante baja (27.2\%). El estudio de Román, Serra-Majem, Ribas-Barba, Pérez-Rodrigo y Aranceta (2008), por ejemplo, informaba de un $43.7 \%$ de los adolescentes que cumplían esta recomendación, porcentaje bastante superior al obtenido en nuestro estudio. Nuestro resultado se situaría por encima del de los norteamericanos (24.2\%) (Mark \& Janssen, 2008) y por debajo del atribuido a los australianos (29-46.6\%) (Hands et al., 2011; Morley, Scully, Dixon \& Wakefield, 2010). Cabe resaltar que mientras que la AAP recomienda un uso no superior a las 2 horas diarias de pantalla (Council on Communication and Media, 2013), el cual incluye TV, ordenador y videojuegos, existen múltiples estudios que han tomado este valor como referencia midiendo únicamente las horas dedicadas a ver la TV, aspecto por el cual dichos trabajos no son comparables con nuestros resultados.

Los resultados de nuestro estudio referentes al UMTP a nivel global en función del sexo muestran una tendencia similar a otros estudios, tanto a nivel nacional (Serrano-Sánchez et al., 2011) como internacional (Ianotti, Kogan, Janssen \& Boyce, 2010) señalando que son los hombres los que les dedican más tiempo que las mujeres. Sin embargo, existen estudios que han encontrado un mayor uso por parte de las mujeres adolescentes (Li et al., 2007) o que no han encontrado diferencias (Sund, Larsson \& Wichstrøm, 2011). Cabe resaltar que en muchos casos, como ocurre en nuestro estudio, las diferencias por sexo se deben, en gran parte, a un mayor uso de los videojuegos por parte de los chicos adolescentes. Como hemos podido observar en nuestra investigación, los adolescentes españoles no mostraban diferencias significativas según el sexo en el tiempo dedicado a ver TV, resultado contrastado por Currie, Gabhainn et al. (2008) y Devís-Devís et al. (2009). No obstante, también hemos encontrado estudios que atribuyen un mayor uso de la TV a las chicas en el caso de adolescentes españoles (Ramos et al., 2012) o mayor uso por parte de los chicos franceses (Dupuy, Godeau, Vignes \& Ahluwalia, 2011).

Si hacemos referencia al uso del ordenador en función del sexo, tampoco en nuestro caso hemos encontrado diferencias. Este patrón de comportamientotambién ha sido confirmado en muestra española(DevísDevís et al., 2009; Serrano-Sánchez et al., 2011). Los resultados referentes a un mayor uso de los videojuegos por parte de los chicos coincide con la literatura científica existente (Ramos et al., 2012; Rey-López et 
al., 2010; Serrano-Sánchez, et al., 2011). Sin embargo, no hemos encontrado ningún estudio que no presente diferencias en función del sexo o que otorgue una mayor dedicación por parte de las chicas.

Centrándonos ahora en la variable curso académico (agrupados en ciclos), veíamos que son los adolescentes de $15-16$ años ( $2^{\circ}$ Ciclo de la ESO) los que obtienen mayores valores medios de UMTP que los más jóvenes (12-14 años, $1^{\text {er }}$ Ciclo de la ESO) y los más mayores (17-18 años, Ciclo de BACH). Un trabajo comparable al de nuestra investigación, y con resultados similares, es el realizado por la Kaiser Foundation Family con adolescentes americanos y en el cual se observaba un mayor uso por parte de los adolescentes de 11 a 14 años en comparación de otros más jóvenes (8-10 años) y más mayores (15-18 años) (Rideout et al., 2010). Sin embargo, otros estudios como el de Kimm et al. (2006), el de Ianotti et al. (2009) (con muestras de adolescentes del Oeste, el Norte y el Sur de Europa) o el de Sund et al. (2011) encontraron un aumento del UMTP proporcional al aumento de la edad. Al contrario, Thibault et al. (2010) no encontraron diferencias de uso en función de la edad. Una posible explicación al patrón de comportamiento encontrado en nuestro estudio estaría relacionada con que los jóvenes de $3^{\circ}$ y $4^{\circ} \mathrm{de}$ ESO pueden disponer de mayor libertad a la hora de utilizar los medios tecnológicos que los adolescentes que cursan $1^{\circ}$ y $2^{\circ}$ de la ESO y, por otra parte, aún no gozan de la autonomía propia de aquellos que cursan el Ciclo de Bachillerato para pasar tiempo fuera del hogar.

Los adolescentes de nuestra muestra no difieren en el tiempo global dedicado al UMTP según su NSE. Únicamente se han visto diferencias en el uso aislado de la TV y del ordenador. Mientras que la TV ya es un medio que poseen prácticamente todos los adolescentes en sus hogares, el ordenador puede estar asociado a familias con mayor estatus socioeconómico, pudiéndoselo permitir únicamente aquellas familias con mayores posibilidades. En nuestra investigación no hemos observado diferencias significativas según el NSE en cuanto al UMTP a nivel global, pero sí existen estudios que han observado una mayor dedicación por parte de aquellos adolescentes que estudian en centros públicos o que poseen un menor NSE (Craig, Mindell \& Hirani, 2009; DevísDevís et al., 2009) o por parte de adolescentes con un NSE más alto (Thibault et al., 2010).

Este estudio presenta varias limitaciones que deben tenerse en cuenta en el momento de valorar sus resultados. En primer lugar se trata de un estudio de diseño transversal que únicamente atiende a comportamientos que pueden darse en un momento dado en los adolescentes participantes, por lo que no pueden derivarse relaciones causales. Por el contrario, un diseño longitudinal permitiría recoger diversas medidas y ver la evolución de las conductas sedentarias, y las asociaciones con el resto de variables, a lo largo de los diferentes cursos académicos. Por otro lado, se ha utilizado un cuestionario de auto-informe para medir las conductas sedentarias, registro que debería acompañarse de métodos de medición objetivos como, por ejemplo, el uso de acelerómetros (Lubans et al., 2011).

\section{Conclusiones e implicaciones futuras}

En conclusión, los adolescentes españoles pasan excesivo tiempo utilizando los medios tecnológicos de pantalla, siendo menos del $30 \%$ los jóvenes que cumplen con la recomendación de no sobrepasar las dos horas diarias de dedicación. Las principales diferencias encontradas muestran un mayor UMTP por parte de los chicos en comparación con las chicas (debido a un mayor uso de los videojuegos) y de los que se encuentran en $2^{\circ}$ Ciclo de ESO en comparación con quienes cursan $1^{\mathrm{er}}$ Ciclo de ESO y el Ciclo de BACH. Pese a las diferencias encontradas en el UMTP a nivel global según las diferentes variables sociodemográficas y el tiempo dedicado de forma aislada a cada uno de los medios estudiados (TV, ordenador y videojuegos), conviene empezar a diseñar estrategias de limitación del tiempo vinculado al uso sedentario de los medios tecnológicos de pantalla en los adolescentes, con especial hincapié en los chicos y en aquéllos que se encuentran a mitad de la adolescencia. Estas estrategias restrictivas han de intentar evitar los largos periodos de tiempo que pasan los y las adolescentes españoles sentados, por los problemas en la salud consecuentes de este estilo de vida (Tremblay et al., 2010). Entre otras estrategias podemos señalar la restricción en el UMTP por parte de los padres y madres, evitar que los jóvenes dispongan de TV u ordenador en sus habitaciones, disminuir los largos periodos que pasan sentados mediante pausas en las que tengan que levantarse (también en la escuela) y fomentar estos hábitos desde el currículo son algunos ejemplos que pueden seguirse para limitar el tiempo sedentario en la adolescencia.

\section{Fuentes de apoyo económico}

La información presentada en este trabajo proviene de un proyecto de investigación I+D+i financiado por el Ministerio de Ciencia e Innovación en 2009 (EDU2009-13664) titulado «Estilo de vida activo, uso de medios tecnológicos y obesidad en adolescentes».

\section{Referencias}

Aires, L., Andersen, L.B., Mendonça, D., Martins, C., Silva, G., \& Mota, J. (2010). A3-year longitudinal analysis of changes in fitness, physical activity, fatness and screen time. Acta Pcediatrica, 99, 140-144. doi: http://dx.doi.org/10.1111/ j.1651-2227.2009.01536.x http://dx.doi.org/10.1111/j.16512227.2009.01536.x

American Academy of Pediatrics Committee on PublicEducation (2001). Children, Adolescents, and Television. Pediatrics, 107(2), 423-426.

Australian Bureau of Statistics. (2006). Children's participation in cultural and leisure activities. Canberra, Australia:ABS.

Beets, M.W., \& Foley, J.T. (2010). Comparison of 3 Different Analytic Approaches for Determining Risk-Related Active and Sedentary Behavioral Patterns in Adolescents. Journal of Physical Activity and Health, 7, 381-392.

Booth, M.L., Okely, A.D., Chey, T., \& Bauman, A. (2002). The reliability and validity of the Adolescent Physical Activity Recall Questionnaire. Medicine \& Science in Sports \& Exercise, 34(12), 1986-1995. doi: http://dx.doi.org/ 10.1097/00005768-200212000-00019

Borraccino, A., Lemma, P., Iannotti, R.J., Zambon, A., Dalmasso, P., Lazzeri, G., ... Cavallo, F. (2009). Socioeconomic Effects on Meeting Physical Activity Guidelines: Comparisons among 32 Countries. Medicine \& Science In Sports \& Exercise, 41(4), 749-756. doi: http://dx.doi.org/10.1249/ MSS.0b013e3181917722

Council on Communication and Media (2013). Children, Adolescents, and the Media. Pediatrics 132(5): 958-61. doi: http://dx.doi.org/10.1542/peds.20132656

Craig, R., Mindell, J., \& Hirani, V. (2009). Health survey for England 2008: physical activity and fitness. Leeds: NHS Information Centre for Health and Social Care.

Currie, C., Gabhainn, S.N., Godeau, E., Roberts, C., Smith, R., Currie, D., ... Barnekow, V. (eds.) (2008). Inequalities in young people's health: HBSC international report from the 2005/06 Survey. Health Policy for Children and Adolescents, 5. Copenhagen: WHO Regional Office for Europe.

Currie, C., Molcho, M., Boyce, W., Holstein, B., Torsheim, T. \& Richter, M. (2008). Researching health inequalities in adolescents: the development of the Health Behaviour in School-Aged Children (HBSC) Family Affluence Scale. Social Science \& Medicine, 66(6), 1429-1436. doi: http://dx.doi.org/10.1016/ j.socscimed.2007.11.024

Del Pozo-Cruz, B., del Pozo-Cruz, J., González, F.J., \& Alfonso, R.M. (2012). Relación entre el nivel de actividad física y sedentarismo, sobrepeso y calidad de vida relacionada con la salud en niños asmáticos en edad escolar: un estudio exploratorio en Sevilla. Retos. Nuevastendencias en Educación Física, Deporte yecreación, 22: 53-56.

Devís-Devís, J., Peiró-Velert, C., Beltrán-Carrillo, V.J., \& Tomás, J.M. (2009). Screen media time usage of 12-16 year-old Spanish school adolescents: Effects of personal and socioeconomic factors, season and type of day. Journal of Adolescence, 32, 213-231. doi: http://dx.doi.org/10.1016/ j.socscimed.2007.11.024

Dewar, D. L., Morgan, P. J., Plotnikoff, R. C., Okely, A. D., Collins, C. E., Batterham, M., ... \& Lubans, D. R. (2013). The nutrition and enjoyable activity for teen girls study: A cluster randomized controlled trial. American Journal of Preventive Medicine, 45(3), 313-317. doi: http://dx.doi.org/10.1016/ j.amepre.2013.04.014

Dumith, S.C., Hallal, P.C., Menezes, A.M.B., \& Araújo, C.L. (2010). Sedentary behavior in adolescents: the 11-year follow-up of the 1993 Pelotas (Brazil) birth cohort study. Cadernos Saúde Pública, 26(10), 1928-1936. doi: http:// dx.doi.org/10.1590/S0102-311X2010001000009 
Dupuy, M., Godeau, E., Vignes, C., \& Ahluwalia, N. (2011). Socio-demographic and lifestyle factors associated with overweight in a representative sample of 1115 year olds in France: Results from the WHO-Collaborative Health Behaviour in School-aged Children (HBSC) cross-sectional study. BMC Public Health, 11, 442. doi: http://dx.doi.org/10.1186/1471-2458-11-442

Encuesta Nacional de Salud de España. (2006). Madrid: Ministerio de Sanidad y Consumo. Consultada el 3dejulio, 2012, en http://www.msc.es/estadEstudios/ estadisticas/encuestaNacional/home.htm

Hancox, R.J., Milne, B.J., \& Poulton, R. (2004). Association between child and adolescent television viewing and adult health: a longitudinal birth cohor study. The Lancet, 364(9430), 257-262. doi: http://dx.doi.org/10.1016/S01406736(04)16675-0

Hands, B.P., Chivers, P.T., Parker, H.E., Beilin, L., Kendall, G., \& Larkin, D. (2011). The associations between physical activity, screen time and weight from 6 to 14 yrs: The Raine Study. Journal of Science and Medicine in Sport, 14, 397-403. doi: http://dx.doi.org/10.1016/j.jsams.2011.03.011

Hardy, L.L., Bass, S.L., \& Booth, M.L. (2007). Changes in sedentary behavior among adolescent girls: a 2.5-year prospective cohort study. Journal of Adolescent Health, 40, 158-165. doi: http://dx.doi.org/10.1016/j.jadohealth.2006.09.009

Hardy, L.L., Booth, M.L., \& Okely, A.D. (2007). The reliability of theAdolescent Sedentary Activity Questionnaire (ASAQ). Preventive Medicine, 45, 71-74. doi: http://dx.doi.org/10.1016/j.ypmed.2007.03.014

Hardy, L.L., Dobbins, T.A., Denney-Wilson, E.A., Okely, A.D., \& Booth, M.L. (2009). Sedentariness, Small-Screen Recreation, andFitness in Youth. American Journal of Preventive Medicine,36(2), 120-125. doi: http://dx.doi.org/10.1016/ j.amepre.2008.09.034

Hernández, J.L., \& Martínez, M.E. (2007). Estilo de vida y frecuencia de práctica de actividad física de la población escolar. En Hernández, J.L., \& Velázquez, R.La educación física, los estilos de viday los adolescentes: cómo son, cómo se ven, quésaben yquéopinan. Barcelona: Graó.

Ho, S.M.Y., \& Lee, T.M.C. (2001). Computer Usage and its relationship with Adolescent Lifestyle in Hong Kong. Journal of Adolescent Health, 29, 258266. doi: http://dx.doi.org/10.1016/S1054-139X(01)00261-0

Ianotti, R.J., Janssen, I., Haug, E., Kololo, H., Annaheim, B., Borraccino, A., \& the HBSC Physical Focus Group. (2009). Interrelationships of adolescent physical activity, screen-based sedentary behaviour, and social and psychological health. International Journal of Public Health, 54, S191-S198.

Ianotti, R.J., Kogan, M.D., Janssen, I., \& Boyce, W.F.(2010). Patterns of Adolescen Physical Activity, Screen-Based Media Use and Positive and Negative Health Indicators in the U.S. and Canada. Journal of Adolescent Health, 44(5), 493499. doi: http://dx.doi.org/10.1016/j.jadohealth.2008.10.142

Kimm, S.Y.S, Glynn, N.W., McMahon, R.P., Voorhees, C.C., Striegel-Moore, R.H., \& Daniels, S.R. (2006). Self-Perceived Barriers to Activity Participation among SedentaryAdolescent Girls. Medicine \& Science in Sports \& Exercise, 38(3), 534-540. doi: http://dx.doi.org/10.1249/01.mss.0000189316.71784.dc

Leatherdale, S.T., \& Ahmed, R. (2011). Screen-based sedentary behaviours among a nationally representative sample of youth: are Canadian kids couch potatoes? Chronic Diseases and Injuries in Canada, 31(4), 141-146.

Li, M.D., Dibley, M.J., Sibbritt, D.W., Zhou, X.B.S., \& Yan, H.M. (2007) Physical Activity and Sedentary Behavior in Adolescents in Xi'an City, China Journal of Adolescent Health, 41, 99-101. doi: http://dx.doi.org/10.1016/ j.jadohealth.2007.02.005

Lubans, D.R., Hesketh, K., Cliff, D.P., Barnett, L.M., Salmon, J., Morgan, P.J., . . Hardy, L.L. (2011). A systematic review of the validity and reliability of sedentary behaviour measures used with children and adolescents. Obesity reviews, 12, 781-799. doi: http://dx.doi.org/10.1111/j.1467789X.2011.00896.x

Mark, A.E., \& Janssen, I. (2008). Relationship between screen time and metabolic syndrome in adolescents. Journal of Public Health, 30(2), 153-160. doi: http: /dx.doi.org/10.1093/pubmed/fdn022

Marshall, S.J., Gorely, T., \& Biddle, S.J.H. (2006). Adescriptive epidemiology of screen-based mediause in youth: Areview and critique. Journal of Adolescence, 29,333-349. doi: http://dx.doi.org/10.1016/j.adolescence.2005.08.016

Moncada, J., \& Chacón, Y. (2012). El efecto de los videojuegos en variables sociales, psicológicas y fisiológicas en niños y adolescentes. Retos. Nuevas tendencias en Educación Física, deporte y recreación, 21, 43-49.

Moreno, M.C., Ramos, P., Rivera, F., Muñoz-Tinoco, V., Sánchez-Queija, I, Granado, M.C., \& Jiménez-Iglesias, A. (2011). Desarrollo adolescentey salud en España. Resumen del estudio Health Behaviour in School Aged Children (HBSC-2006). Ministerio de Sanidad, Política social e Igualdad. Madrid. Consultadoel 7 deabrilde2012 enhttp://www.msc.es/profesionales/saludPublica/ prevPromocion/promocion/saludJovenes/estudioHBSC/ ResumenHBSC2006.htm

Morley, B., Scully, M., Dixon, P., \& Wakefield, M. (2010). National Secondary Students Diet and Activity Survey, 2009-2010: Main Report. Melbourne: Cancer CouncilVictoria. Disponibleen:http://www.cancervic.org.au/module_research/ module_research_projects/secondary-students-diet-nassda.html
Nihill, G. F. J., Lubans, D. R., \& Plotnikoff, R. C. (2012). Associations between sedentary behavior and self-esteem in adolescent girls from schools in lowincome communities. Mental Health and Physical Activity. 6(1):30-35. doi: http://dx.doi.org/10.1016/j.mhpa.2012.02.003

Norman, G.J., Schmid, B.A., Sallis, J.F., Calfas, K.J., \& Patrick, K. (2005). Psychosocial and Environmental Correlates of Adolescent Sedentary Behaviors. Pediatrics, 116, 908-916. doi: http://dx.doi.org/10.1542/peds.2004-1814

Nuviala,A., Munguía, D., Fernández,A., Ruiz, F., \& García, M.E.(2009).Typologies of occupation of leisure-time of Spanish adolescent. The case of the participants in physical activities organized. Journal of Human Sport and Exercise, 4(1), 29-39. doi: http://dx.doi.org/10.4100/jhse.2009.41.04

Pate, R.R., Colabianchi, N., Porter, D., Almeida, M.J., Lobelo, F., \& Dowda, M. (2008). Physical Activity and Neighborhood Resources in High School Girls. American Journal of Preventive Medicine,34(5), 413-419. doi: http://dx.doi.org/ 10.1016/j.amepre.2007.12.026

Patriarca, A., Di Giuseppe, G., Albano, L., Marinelli, P., \& Angelillo, I.F. (2009). Use of television, videogames, and computer among children and adolescents in Italy. BMC Public Health, 9, 139. doi: http://dx.doi.org/10.1186/14712458-9-139

Ramos, P., Rivera, F., Moreno, C., \& Jiménez-Iglesias, A. (2012). Análisis de clúster de la actividad física y las conductas sedentarias de los adolescentes españoles, correlación con la salud biopsicosocial. Revista de Psicología del Deporte, 21(1), 99-106.

Rey-López, J.P., Tomas, C., Vicente-Rodríguez, G., Gracia-Marco, L., JiménezPavón, D., Pérez-Llamas F., ... the AVENA Study Group. (2010). Sedentary behaviours and socio-economic status in Spanish adolescents: the AVENA study. European Journal of Public Health, 21(2), 151-157.doi: http://dx.doi.org/ 10.1093/eurpub/ckq035

Rideout, V.J., Foehr, U.G., \& Roberts, D.F. (2010). Generation M². Media in the Lives of 8- to 18-Year-Olds. AKaiser Family Foundation Study. Menlo Park, California.

Román, B., Serra-Majem, L., Ribas-Barba, L., Pérez-Rodrigo, C., \& Aranceta, J. (2008). How many children and adolescents in Spain comply with the recommendations on physical activity? Journal of Sports Medicine and Physical Fitness, 48(3), 380-387.

Romero, I., Mendonça, M.A., Almeida, R., Almeida, B., de Oliveira, V., \& Camargo,A.C.(2011).AtividadeFísica, Horas de AssistênciaàTV eComposição Corporal em Crianças e Adolescentes. Arquivos Brasileiros de Cardiologia, 95(2), 159-165.

Serra, L., Ribas, L., Aranceta, J., Pérez, C., Saavedra, P., \& Peña, L. (2003). Obesidad infantil y juvenil en España. Resultados del Estudio enKid (19982000). Medicina Clínica, 121(19), 725-732.

Serrano-Sánchez, J.A., Martí-Trujillo, S., Lera-Navarro, A., Dorado-García, C., González-Henríquez, J.J., \& Sanchís-Moysi, J. (2011). Associations between Screen Time and Physical Activity among Spanish Adolescents. PLoS One, 6(9), e24453. doi: http://dx.doi.org/10.1371/journal.pone.0024453

Sund, A.M., Larsson, B., \& Wichstrøm, L. (2011). Role of physical and sedentary activities in the development of depressive symptoms in early adolescence. Social Psychiatry and Psychiatric Epidemiology, 46, 431-441. doi: http:// dx.doi.org/10.1007/s00127-010-0208-0

Te Velde, S.J., De Bourdeaudhuij, I., Thorsdottir, I., Rasmussen, M., Hagströmer, M., Klepp, K., \& Brug, J. (2007). Patterns in sedentary and exercise behaviors and associations with overweight in 9-14-year-old boys and girls - a crosssectional study. BMC Public Health, 7, 16.

The Sedentary Behaviour and Obesity Expert Working Group. (2010). Sedentary Behaviour and Obesity: Review of the Current Scientific Evidence. Disponible en: http://www.dh.gov.uk/prod_consum_dh/groups/dh_digitalassets/ documents/digitalasset/dh_128225.pdf

Thibault, H., Contrand, B., Saubusse, E., Baine, M., \& Maurice-Tison, S. (2010). Risk factors for overweight and obesity in French adolescents: Physical activity, sedentary behavior and parental characteristics. Nutrition, 26, 192-200. doi: http://dx.doi.org/10.1016/j.nut.2009.03.015

Tremblay, M.S., Colley R.C., Saunders, T.J., Healy, GN., \& Owen, N. (2010). Physiological and health implications of a sedentary lifestyle. Applied Physiology, Nutrition, and Metabolism, 35, 725-740. doi: http://dx.doi.org/10.1139/ H10-079

Tukey, J. (1977). Exploratory Data Analysis. Reading. Mass.: Addison-Wesley.

Vicente-Rodríguez, G., Rey-López, J.P., Martín-Matillas, M., Moreno, L.A., Wärnberg, J. Redondo, C., ... the AVENA Study Group. (2008). Television watching, videogames, and excess of body fat in Spanish adolescents: The AVENA study. Nutrition, 24, 654-662. doi: http://dx.doi.org/ 10.1016\%2Fj.nut.2008.03.011

Von Rueden, U., Gosch, A., Rajmil, L., Bisegger, C. \& Ravens-Sieberer, U. (2006). Socioeconomic determinants of health related quality of life in childhood and adolescence: results from a European study. Journal of Epidemiology \& Community Health, 60(2), 130-135. doi: http://dx.doi.org/10.1136/ jech.2005.039792 